


\title{
REPRESENTATIVIDAD DE LAS MUJERES EN LOS ORGANISMOS DE COGOBIERNO DE LAS INSTITUCIONES DE EDUCACIÓN SUPERIOR DE ECUADOR. EL CASO DE LA UNIVERSIDAD LAICA ELOY ALFARO DE MANABÍ
}

\section{REPRESENTATION OF WOMEN IN THE ORGANIZATIONS OF COGOBIERNO OF THE INSTITUTIONS OF HIGHER EDUCATION OF ECUADOR. THE CASE OF THE UNIVERSITY LAICA ELOY ALFARO DE MANABÍ}

\author{
JHESENIA SACOTO LOOR ${ }^{* 1}$ \& MERCEDES OSUNA RODRIGUEZ ** \\ UNIVERSIDAD LAICA ELOY ALFARO DE MANABÍ (ECUADOR)* \\ UNIVERSIDAD DE CÓRDOBA (ESPAÑA) $)^{* *}$
}

\section{RESUMEN}

La representatividad de las mujeres en las instituciones de Educación Superior subyace en los logros de los movimientos feministas durante los años 70 por la participación de las mujeres en el ámbito público. El presente estudio analiza la representatividad de las mujeres docentes, con una muestra de 341 mujeres docentes, cuya información fue obtenida del Sistema Integrado de Información de Talento Humano, que participan en varias funciones de cogobierno y direcciones departamentales administrativas y académicas de la Universidad Laica Eloy Alfaro de Manabí (ULEAM). Los resultados muestran que a pesar de que existen normativas que garantizan la igualdad de género en la práctica no se cumple, pues la representatividad de la mujer en la ULEAM es del $9.3 \%$ de acuerdo a la población de mujeres docentes (341). Se recomienda indagar en los factores que impiden la aplicación efectiva de la política pública e interna de la institución respecto a representatividad y principio de igualdad de género.

Palabras claves: representatividad de mujeres; educación superior; igualdad de género; participación.

\begin{abstract}
The representativeness of women in higher education institutions underlies the achievements of feminist movements during the 70s by the participation of women in the public sphere. The present study analyzes the representation of women teachers, with a sample of 341 women teachers, whose information was obtained from the Integrated Human Talent Information System, participating in various co-government functions and administrative and academic departmental directions of the University Laica Eloy Alfaro de Manabi (ULEAM). The results show that although there are regulations that guarantee gender equality in practice, it is not fulfilled, since the representativeness of women in ULEAM is $9.3 \%$ according to the population of women teachers (341). It is recommended to investigate the factors that impede the effective application of the public and internal policy of the institution regarding representativeness and the principle of gender equality.
\end{abstract}

Key words: representation of women; higher education; gender equality; participation.

\footnotetext{
1 Email: z62salor@uco.es
} 


\section{Introducción}

La igualdad de género es uno de los retos más importantes en la Educación Superior, porque requiere de una comprensión desde el enfoque de derechos, como es erradicar la desigualdad de género establecido en los compromisos adquiridos durante la Conferencia Mundial de Beijing (Organización de las Naciones Unidas, 1995). A medida que la mujer ha empezado un proceso de empoderamiento, como sujeto de derechos, y ejercer su derecho a la educación, ha asumido retos de llegar a puestos representativos en cualquier ámbito donde se ha profesionalizado (Kabeer, 2001)

Según el estudio sobre Igualdad de Género y Educación Superior: Retos por alcanzar en la Unión Europea de Matarranz \& Ramírez (2018) se concluyen que, a pesar de los avances en el acceso a la educación, aprobación de políticas en relación a la igualdad de género, aún la representatividad de las mujeres en máximos cargos en instituciones de Educación Superior es baja. Estudios realizados en varios países de América Latina, sobre representatividad de las mujeres (Pérez, 2018) en cargos directivos en las instituciones de Educación Superior (Allison, Gregoratti, \& Tornhill, 2019), concluyen que representatividad siguen siendo limitada y con desigualdades salariales (Bolaños, 2005) en puestos directivos entre hombres y mujeres.(Ordorika, 2015).

En Ecuador, desde la Constitución del 2008 se declara el principio de igualdad y derechos de participación que establecen que la ciudadanía podrá desempeñar empleos y funciones públicas con base en méritos y capacidades, y en un sistema de selección y designación transparente, incluyente, equitativo, pluralista y democrático, que garantice su participación, con criterios de equidad y paridad de género, igualdad de oportunidades para la personas con discapacidad y participación intergeneracional. A su vez, todas las instituciones de Educación Superior debían reformar sus normativas internas que garantizaren el cumplimiento de los derechos, mediante la aplicación de políticas internas.

La reforma del estatuto de la Universidad Laica Eloy Alfaro de Manabí 2014 (ULEAM) establece en su Art. 7, que el cogobierno, consiste en la dirección compartida por parte de los diferentes sectores de la comunidad de la institución, docentes, estudiantes y funcionarios acorde a principios de calidad, igualdad de oportunidades, alternabilidad y equidad de género (Consejo Universitario, 2014).

En tal razón la institución cuenta con varios espacios de cogobierno en el que participan representantes de autoridades, docentes, estudiantes y funcionarios para la toma de decisiones que beneficien a toda la institución. Además cuenta con espacios de apoyo, asesoría, en donde resuelven o proponen al Consejo Universitario, como Consejos y Comisiones; también, según el organigrama de la institución se encuentran Departamentos Centrales de Coordinación Académica y Administrativa, que son: Admisión y Nivelación Universitaria, Planeamiento Académico, Investigaciones, Promoción y Desarrollo Cultural, Evaluación Interna, Información Bibliográfica y Servicios Educativos, Bienestar Estudiantil, Técnico, Consultoría y Asesoría Jurídica, Vinculación con la Colectividad, de Relación y Cooperación Internacionales y de Edición y Publicaciones, Medio Ambiente, Seguridad y Gestión de Riesgos. A nivel de facultades existen organizacionalmente Decanatos y Coordinaciones de Carrera; espacios que administran académicamente la educación en la ULEAM.

El presente estudio analiza la representatividad de las docentes en espacios de tomas de decisiones de la Universidad Laica Eloy Alfaro de Manabí (ULEAM) durante el periodo 2016-2018 a partir de la información obtenida del Sistema Integrado de Información de Talento Humano de esta institución y nos ha permitido analizar la frecuencia y el porcentaje de mujeres docentes que participan como miembros en los distintos órganos de cogobierno de esta universidad. 


\section{Marco teórico}

\subsection{Marco normativo sobre la igualdad de género en Educación Superior}

La igualdad de género nace como un principio y derecho evocado por los grupos de movimientos de mujeres para romper un sistema patriarcal que ha discriminado, violentado y oprimido a las mujeres durante siglos y que busca la armonía entre hombres y mujeres en igualdad de oportunidades y condiciones tanto el ámbito privado como en el público (Melero, 2010).

En Ecuador desde el año 2010 inició un proceso de cambios en todas las normativas, incluyendo las de Educación Superior, estos cambios asumen compromisos internacionales que garantizan el cumplimiento de los derechos:

A nivel internacional los compromisos suscritos por Ecuador y ratificados fueron: La Cuarta Conferencia Mundial sobre la mujer (Organización de las Naciones Unidas, 1995); la Convención sobre la Eliminación de todas las formas de discriminación contra la mujer (Committe on the Elimination of Discrimination against Women, 2006); la Cumbre del Milenio (Naciones Unidas, 2015) en el que estableció 8 objetivos, de los cuales tres se refieren a la igualdad entre los géneros, autonomía de la mujer, reducción de la mortalidad en la infancia y al mejoramiento de la salud materna.

Estos compromisos internacionales asumidos y reflejados en la Constitución Política del Ecuador (Tribunal Constitucional del Ecuador, 2008) fueron el resultado de la incidencia política de las organizaciones de las mujeres, presentes en el país desde el inicio de la democracia en el año de 1979, posteriormente en los años 80 y 90 se logra instaurar el análisis de la violencia intrafamiliar y de género como un problema para lograr la igualdad y la no discriminación. Producto del profundo análisis se aprueba y divulga la Ley de Maternidad Gratuita y Atención a la Infancia (Congreso Nacional, 2006) y la conformación del Consejo Nacional de las Mujeres CONAMU, como organismo máximo adscripto a la Vicepresidencia de la República. El hecho de contar con este organismo, permitió la lucha constante en el Congreso Nacional, en la promulgación de nuevas leyes a favor de las mujeres a finales de los años 90, como la Ley de Amparo Laboral de la Mujer (Congreso Nacional, 2000), obligando que existiera al menos el $20 \%$ de mujeres en las Cortes Superiores de Justicia, Juzgados, Notarías y Registros. (Palacios, 2008)

A partir de los procesos electorales del 2007, en los que se impulsa la reforma de la Constitución del Ecuador, los movimientos de mujeres se activaron para plantear un conjunto de propuestas que no serían objeto de negociación a los derechos ya adquiridos en los años 90. Los derechos por los cuales las mujeres estaban dispuestas a mantenerse en lucha estaban relacionados con:

- Derecho a la libertad de conciencia y a adoptar decisiones.

- Derecho a la igualdad real o material en la aplicación de medidas de acción positiva.

- Derecho a tomar decisiones libres y responsables sobre su cuerpo, vida sexual y reproducción.

- Derecho a la paridad, en la representación de mujeres y hombres en las funciones del Estado, organismos de control, régimen autónomo, gobiernos seccionales, cargos públicos y de elección popular.

- Derecho a la justicia a víctimas de abuso sexual y violencia de género.

- Erradicación del sexismo, machismo, androcentrismo y prácticas discriminatorias.

- Prohibición a los hombres, quienes adeuden pensiones alimenticias o sean responsables de violencia sexuales o de género.

- Conciliación a la labor productiva con la reproductiva (Reimberto, 2008).

Los logros alcanzados por las mujeres en la Constitución Política del Ecuador del 2008, están relacionados a los principios de democracia, igualdad, no-discriminación, respeto a la diversidad, estado laico, pluriculturalidad, ejercicio y exigibilidad pleno de los derechos sexuales y reproductivos, establecidos en los Capítulos primero, segundo, quinto, sexto y octavo del Título II 
sobre derechos constitucionales; economía soberana y solidaria, justicia de género, expresados en el Titulo III, sobre garantías constitucionales; y paridad, participación en el poder público y ciudadano e institucionalidad para la igualdad de mujeres, declarados en el Título IV sobre participación y organización del poder (Tribunal Constitucional del Ecuador, 2008).

Lo anterior expuesto es reflejado en la Ley Orgánica de Educación Superior ecuatoriana (Consejo de Educación Superior, 2018), dentro de los principios hace referencia a la igualdad de oportunidades expresados en el Art.12, y a su vez en el Art. 71 garantiza a todos los actores del sistema de educación superior iguales oportunidades (...) sin discriminación de género, credo, orientación sexual, etnia, cultura, preferencia política, condición socioeconómica o discapacidad.

Institucionalmente, la ULEAM, reorienta su estatuto y establece en el Art. 4, de los Objetivos y Estrategias Institucionales "Garantizar la accesibilidad a la educación superior, sin discriminación a través de un proceso transparente e igualitario de admisión y nivelación tomando en consideración aptitudes y conocimientos del aspirante, de conformidad con las regulaciones de la Secretaria Nacional de Ciencia y Tecnología (SENESCYT)" (Herdoíza, 2015).

Respecto al Art. 7 de la misma normativa en su Principio de Cogobierno: El cogobierno es parte circunstancial de la autonomía universitaria responsable. Consiste en la dirección compartida por pares de los diferentes sectores de la comunidad de la institución, profesores/as estudiantes, empleados y trabajadores acorde con los principios de calidad, igualdad de oportunidades alternabilidad y equidad de género. Además, en el Art. 10 se garantiza la participación de docentes, trabajadores y estudiantes en la conformación de los organismos colegiados, respetando la paridad de género, igualdad de oportunidades y sin discriminación alguna. Estatuto ULEAM (2014).

\subsection{La representatividad en espacios de cogobierno en instituciones de Educación Superior.}

El término representatividad subyace en los movimientos feministas durante los años 70 por la participación de las mujeres en el ámbito público (Luna, 1998). El presente estudio analiza la representatividad de las mujeres docentes en las funciones directivas en espacios de tomas de decisiones, como avance de la participación de las mujeres en espacios en el ámbito público en el sistema de Educación Superior.

Durante siglos las mujeres han luchado por visibilizar sus capacidades y profesionalismo en el desempeño de las funciones que realizan, en especial en cargos directivos en las universidades; y éste, ha sido uno de los retos más grandes contra el sistema patriarcal (Luna, 1998); en efecto, ha existido un aumento notable de participación de las mujeres, a nivel de Europa, y América Latina. (Zabludovsky, 2007). En el caso de Ecuador, la participación ha ido creciendo paulatinamente al cumplimiento de las políticas públicas en todas las instituciones que deben elegir a sus autoridades de manera democrática, respetando el principio de igualdad de género y paridad en la conformación de partidos electorales; sin embargo en las universidades, se evidencia un fenómeno, que desde la aprobación de la Constitución ecuatoriana actual, las listas de elección de autoridades universitarias están conformadas por hombres y mujeres, pero la figura de autoridad máxima en la mayor parte de los casos es asumida por un hombre (Observación personal). Se evidencia la falta de igualdad de oportunidades a mujeres y una modesta tendencia a la paridad reflejado en la representación femenina en carreras dedicadas a la educación y a los cuidados (Matarranz \& Ramírez, 2018). En el caso de Ecuador, la representatividad en las instituciones de Educación Superior está establecida en la Ley Orgánica de Educación Superior (2010), lo cual debe cumplir con el análisis de los perfiles académicos, científicos y experienciales, para asumir funciones de rectoría, vicerrectoría, decanatos, direcciones departamentales administrativas y académicas, en cuyas elecciones y designaciones, bajo el principio de igualdad de género y paridad, se debe garantizar la igualdad de oportunidades. 


\section{Metodología}

\subsection{Diseño}

El método utilizado en esta investigación se basa en una metodología transversal cuantitativa de corte descriptivo y comparativo.

\subsection{Objetivo}

Analizar la representación de las docentes en los órganos de cogobierno, direcciones, decanatos y coordinaciones académicas de la Universidad Laica Eloy Alfaro de Manabí (ULEAM).

\subsection{Participantes}

La muestra estuvo formada por 341 mujeres docentes de la Universidad Laica Eloy Alfaro de Manabí (ULEAM), pertenecientes a 19 titulaciones de las distintas facultades integradas en esta universidad. En la tabla siguiente (Tabla 1) podemos observar que el mayor número de mujeres docentes se encuentran en la titulación de Ciencias médicas (20.5\%), seguido de la titulación de Ciencias de la Educación con un 10\%. Sin embargo, las titulaciones en las que se encuentra una menor presencia de mujeres docentes son, la titulación de Ciencias Informáticas (1.2) seguida de la titulación de Arquitectura con un 1.5\% e Ingeniería Industrial con un 1.8\%. La información ha sido extraída del Sistema Integrado de Talento Humano de la ULEAM.

Tabla 1.

Distribución de la muestra por titulaciones

\begin{tabular}{lcc}
\hline \multicolumn{1}{c}{ Titulaciones } & f & \% \\
\hline Arquitectura & 5 & 1.5 \\
Ciencias Administrativas & 27 & 7.9 \\
Ciencias Agropecuarias & 15 & 4.4 \\
Ciencias de la Comunicación & 17 & 5 \\
Ciencias de la Educación & 34 & 10 \\
Ciencias del Mar & 8 & 2.3 \\
Ciencias Económicas & 19 & 5.6 \\
Ciencias Informáticas & 4 & 1.2 \\
Ciencias Médicas & 70 & 20,5 \\
Contabilidad y Auditoria & 12 & 3.5 \\
Derecho & 8 & 2.3 \\
Enfermería & 17 & 5 \\
Gestión, Desarrollo y Secretariado Ejecutivo & 23 & 6.7 \\
Hotelería y Turismo & 18 & 5.3 \\
Ingeniería & 11 & 3.2 \\
Ingeniería Industrial & 6 & 1.8 \\
Odontología & 15 & 4.4 \\
Psicología & 15 & 4.4 \\
Trabajo Social & 17 & 5 \\
\hline
\end{tabular}

\subsection{Procedimiento}

Se analizó la representación de las docentes en la toma de decisiones y organización institucional durante el periodo del 2017-2018 en la Universidad Laica Eloy Alfaro de Manabí (ULEAM), Consejo Universitario, Decanatos, Direcciones Departamentales, Coordinaciones de 
carrera y Representantes de Docentes al Consejo Universitario. El análisis se realizó a partir de los datos disponibles en el Sistema Integrado de Información de Talento Humano de la ULEAM. Se realizaron análisis descriptivos y comparativos para conocer el nivel de representación de las mujeres docentes en cada titulación durante el periodo 2017-2018 en la Universidad Laica Eloy Alfaro de Manabí (ULEAM).

\section{Resultados}

Según la muestra tomada del Sistema Integrado de Información de Talento Humano, 341 mujeres laboran en 19 titulaciones desarrollando diferentes funciones, docencia, investigación, vinculación, actividades administrativas y representativas según su nominación. El siguiente estudio muestra los resultados de las mujeres docentes que realizan funciones representativas en espacios donde toman decisiones que atañen al cumplimiento de la política pública y a los derechos de quienes laboran en esta entidad de Educación Superior. A continuación, se presenta la distribución estadística de las docentes por titulación y la representatividad en diferentes funciones que serán descritos a continuación: El Consejo Universitario (CU) es el principal órgano y autoridad máxima que rige la ULEAM y está formado por los Vicerrectores/as (V), Decanos/as (D) y Representantes Docentes (RD) sumando un total de 45 participantes, de los cuales 12 son mujeres docentes lo cual representa el $26,6 \%$, en este espacio. Esto equivale a 3,5\% de Representatividad de Mujeres Docentes (RMD) en relación con la muestra de nuestro estudio (Tabla 2).

Tabla 2.

Representación de Mujeres Docentes (RMD) en el Consejo Universitario (CU)

\begin{tabular}{lcccc}
\hline \multicolumn{1}{c}{ Titulaciones } & V & D & RD & RMD \% \\
\hline Arquitectura & 0 & 0 & 0 & 0 \\
Ciencias Administrativas & 0 & 0 & 1 & 3.7 \\
Ciencias Agropecuarias & 0 & 0 & 0 & 0 \\
Ciencias de La Comunicación & 0 & 0 & 0 & 0 \\
Ciencias de La Educación & 1 & 2 & 1 & 11.8 \\
Ciencias del Mar & 0 & 0 & 0 & 0 \\
Ciencias Económicas & 1 & 0 & 1 & 10.5 \\
Ciencias Informáticas & 0 & 1 & 0 & 25 \\
Ciencias Médicas & 0 & 0 & 0 & 0 \\
Contabilidad y Auditoria & 0 & 0 & 0 & 0 \\
Derecho & 0 & 0 & 0 & 0 \\
Enfermería & 0 & 1 & 0 & 5.9 \\
Gestión, Desarrollo y Secretariado Ejecutivo & 0 & 1 & 0 & 4.4 \\
Hotelería y Turismo & 0 & 0 & 0 & 0 \\
Ingeniería & 0 & 0 & 0 & 0 \\
Ingeniería Industrial & 0 & 0 & 0 & 0 \\
Odontología & 0 & 0 & 0 & 0 \\
Psicología & 0 & 1 & 1 & 13.3 \\
Trabajo Social & 0 & 0 & 0 & 0 \\
\hline & 2 & 6 & 4 & 3.5 \\
\hline
\end{tabular}


La categoría de Consejos y Comisiones Permanentes no se consideró en el análisis estadístico debido a la existencia de una doble función al ser también integrantes del Consejo Universitario.

La ULEAM tiene en su organigrama institucional un total de 10 Departamentos Centrales de Coordinación Académica y Administrativa: Planeamiento Académico, Investigaciones, Promoción y Desarrollo Cultural, Evaluación Interna, Información Bibliográfica y Servicios Educativos, Bienestar Estudiantil, Técnico, Vinculación con la Colectividad, de Relación y Cooperación Internacionales y de Edición y Publicaciones, Gestión Ambiental, y Seguridad y Riesgos. La representatividad de mujeres docentes es de $50 \%$ en relación al número de departamentos que existen en la institución. De acuerdo a este resultado, la representatividad respecto a la población de mujeres docentes es del $1.5 \%$ (Tabla 3 ).

Tabla 3.

Representación de Mujeres Docentes (RMD) en Direcciones Departamentales (DD)

\begin{tabular}{lccc}
\hline \multicolumn{1}{c}{ Titulaciones } & DD & f & RMD \% \\
\hline Arquitectura & 0 & 0 & 0 \\
Ciencias Administrativas & 1 & 1 & 3.7 \\
Ciencias Agropecuarias & 1 & 0 & 6.6 \\
Ciencias de la Comunicación & 0 & 0 & 0 \\
Ciencias de la Educación & 0 & 0 & 0 \\
Ciencias del Mar & 0 & 0 & 0 \\
Ciencias Económicas & 0 & 0 & 0 \\
Ciencias Informáticas & 0 & 0 & 0 \\
Ciencias Médicas & 0 & 0 & 0 \\
Contabilidad y Auditoria & 1 & 1 & 8.3 \\
Derecho & 0 & 0 & 0 \\
Enfermería & 0 & 0 & 0 \\
Gestión, Desarrollo y Secretariado Ejecuti & 1 & 1 & 4.3 \\
Hotelería y Turismo & 1 & 1 & 5.5 \\
Ingeniería & 0 & 0 & 0 \\
Ingeniería Industrial & 0 & 0 & 0 \\
Odontología & 0 & 0 & 0 \\
Psicología & 0 & 0 & 0 \\
Trabajo Social & 0 & 0 & 0 \\
\hline & 5 & 5 & 1.5 \\
\hline
\end{tabular}

La institución cuenta con 19 titulaciones, asumidos por decanatos, de los cuales existen 8 mujeres asumiendo esta responsabilidad, lo que representa el $42 \%$ del total de decanatos; 13 mujeres coordinadoras de 19 titulaciones, que equivale al 68\%. Desde el punto de la representatividad institucional corresponde al $6.2 \%$ (Tabla 4 ). 
Tabla 4.

Representación de mujeres en Decanatos (D) y Coordinaciones Académicas (CA).

\begin{tabular}{lccc}
\hline \multicolumn{1}{c}{ Titulaciones } & D & CA & RMD\% \\
\hline Arquitectura & 0 & 0 & 0 \\
Ciencias Administrativas & 0 & 0 & 0 \\
Ciencias Agropecuarias & 1 & 1 & 13.3 \\
Ciencias de La Comunicación & 0 & 1 & 5.9 \\
Ciencias de La Educación & 2 & 2 & 11.8 \\
Ciencias del Mar & 0 & 0 & 0 \\
Ciencias Económicas & 0 & 1 & 5.3 \\
Ciencias Informáticas & 1 & 0 & 25 \\
Ciencias Médicas & 0 & 2 & 2.9 \\
Contabilidad y Auditoria & 0 & 0 & 0 \\
Derecho & 0 & 1 & 12.5 \\
Enfermería & 1 & 1 & 11.8 \\
Gestión, Desarrollo y Secretariado Ejecutivo & 1 & 2 & 13 \\
Hotelería y Turismo & 0 & 0 & 0 \\
Ingeniería & 0 & 0 & 0 \\
Ingeniería Industrial & 0 & 0 & 0 \\
Odontología & 0 & 1 & 6.7 \\
Psicología & 1 & 1 & 13.3 \\
Trabajo Social & 1 & 0 & 5.9 \\
\hline & 8 & 13 & 6.2 \\
\hline
\end{tabular}

Los resultados antes expuestos sobre el Cogobierno, Direcciones Departamentales, Decanatos y Coordinaciones indican que 32 mujeres docentes participan de las diferentes funciones, lo que en representatividad de mujeres docentes alcanza el $9.3 \%$ de la muestra poblacional de mujeres docentes (341) que laboran en la ULEAM.

\section{Discusión}

La Universidad Laica Eloy Alfaro de Manabí (ULEAM), regula la normativa de acuerdo a los cambios establecidos en la Constitución de la República del Ecuador(Tribunal Constitucional del Ecuador, 2008), en la que se prioriza diferentes enfoques y principios, entre ellos la igualdad de género, derechos a la educación, de participación (...); esto respondiendo a su vez a las exigencias nacionales que vayan de acuerdo al Plan Nacional para el Buen Vivir (2013-2017) (Consejo Nacional de Planificación, 2013), documento que regula la planificación de todas las instituciones del Estado ecuatoriano. Por esta razón, la ULEAM, desde año 2014 aprueba un nuevo Estatuto acorde a las normativas que impera en el Ecuador.

De acuerdo a lo anterior, el presente estudio analiza la representatividad de las docentes en los órganos de cogobierno, direcciones departamentales, decanatos y coordinaciones académicas de la ULEAM. Los resultados han demostrado que la participación de las mujeres docentes en el Consejo Universitario corresponde a un tercio en esta instancia; dado a lo establecido en la Constitución del Ecuador que orienta la participación bajo los principios de igualdad, un sistema de selección y designación transparente, incluyente, equitativo, pluralista y democrático, que garantice su participación, con criterios de equidad y paridad de género, igualdad de oportunidades 
para las personas con discapacidad y participación intergeneracional.(Tribunal Constitucional del Ecuador, 2008), todo esto coherente con lo estipulado en el Estatuto de la ULEAM en el que garantiza la igualdad de género (Consejo Universitario, 2014). Sin embargo, analizando la normativa sobre la estructuración de este órgano, la institución debe garantizar que la selección de los decanatos sea paritaria, debido a que es la rectoría del establecimiento quien selecciona al fiel cumplimiento de perfiles que puedan asumir el cargo. Por otro lado, las representaciones de profesorado deberán garantizar la paridad de género y la igualdad de oportunidades desde las facultades, que son los espacios de elección. Los resultados revelados en este estudio, son similares a investigaciones realizadas en Europa (Peterson, 2017) y algunos países de América Latina, en donde se evidencia que $6.25 \%$ se encuentran en cargos directivos 0 de representatividad (Ordorika, 2015), similar al resultado que muestra la ULEAM en relación a la representatividad de las mujeres docentes en espacios de toma de decisiones de acuerdo población total de docentes que es $9.3 \%$, por lo que se puede determinar que es bajo.

La ULEAM tiene en su organigrama institucional un total de 10 Departamentos Centrales de Coordinación Académica y Administrativa, la representatividad de mujeres docentes es de $50 \%$ en relación al número de departamentos que existen en la institución. Desde la primicia de la paridad, se puede determinar que se cumple con lo establecido en la normativa institucional; sin embargo, estos espacios de acuerdo a este resultado, la representatividad de acuerdo a la población de mujeres docentes es del $1.21 \%$ se considera baja, sin embargo de acuerdo al número de departamentos que existen en la institución no lo es, por lo que se podría ver como un avance en temas de igualdad; cabe recalcar que estos departamentos únicamente toman decisiones respecto a las resoluciones que el Consejo Universitario, lo cual comprueba una vez que el CU es el organismo donde debe primar un espacio paritario de participación de mujeres en las tomas de decisiones, lo cual es un gran hallazgo de este estudio.

La institución cuenta con 19 titulaciones, representados por decanatos, de los cuales el $42 \%$ son mujeres; estos datos son ligeramente más altos respecto al estudio sobre las mujeres en México: trabajo, Educación Superior y esferas de poder, en el que evidencia que las mujeres representan el $35,5 \%$ de trabajo y representatividad en espacios de poder en países de América Latina (Zabludovsky, 2007). Las titulaciones donde la mayoría de las decanas se encuentran como representantes, son aquellas feminizadas (Navarro, Ramos, \& Cejas, 2018), como Educación, Enfermería, Trabajo Social, Psicología, Gestión, Desarrollo y Secretariado Ejecutivo; a excepción de Ciencias Informáticas. Es importante mencionar que la revisión del Sistema de Información de Talento Humano muestra la existencia de mujeres con perfiles que podrían permitirles asumir decanatos en las Facultades de Ciencias duras y experimentales; esto es un hallazgo importante, debido a que es necesario estimar qué condiciones existen a nivel institucional para elegir a mujeres que representen estas áreas; un estudio similar sobre Mujeres y Ciencia: Modelos de Participación explica que es de muy poco prestigio para las instituciones de Educación Superior que las mujeres puedan asumir la dirección de esas áreas (Ruzzeddu, 2019), lo cual odría convertirse en un estereotipo de quien está a cargo de la rectoría de las instituciones en pensar que una mujer no es capaz de asumir un decanato en áreas de las ciencias duras y experimentales, estos datos son parecidos al estudio realizado por Matarranz \& Ramírez, (2018). En relación a las coordinaciones académicas que se encuentran en cada facultad bajo el organigrama del decanato, el 68\% están representadas por docentes mujeres. Desde el contexto de la representatividad institucional corresponde al $6.2 \%$, que es el punto más alto en relación con el cogobierno y direcciones departamentales.

A modo de conclusión de esta investigación se determina que a nivel global en la ULEAM la representatividad de la mujer es del $9.3 \%$, lo cual sigue siendo un bajo porcentaje, sin embargo existen paridades en representaciones departamentales donde se toman decisiones en función de procesos administrativos y de resoluciones del Consejo Universitario lo cual podría no ser significativo para la institución, también una aparente paridad en la representaciones de decanatos 
y las coordinaciones académicas. Es recomendable que para posteriores estudios se indague sobre los factores que impidan la aplicación efectiva de la política pública e interna de la institución respecto a las elecciones de representantes en función del principio de igualdad.

\section{Referencias bibliográficas}

Allison, K., Gregoratti, C., \& Tornhill, S. (2019). From the Academy to the Boardroom: Methodological Challenges and Insights on Transnational Business Feminism. Feminist Review, 121(1), 53-65. doi: 10.1177/0141778918817739

Bolaños, C. (2005). La calidad universitaria desde una perspectiva de género. Actualidades Investigativas En Educación, 5, 1-15.

Committe on the Elimination of Discrimination against Women. (2006). Convention on the Elimination of All Forms of Discrimination against Women Committee on the Elimination of Discrimination against Women Ways and means of expediting the work of the Committee on the Elimination of Discrimination against Women. Recuperado de http://www.bayefsky.com/general/cedaw_c_2006_ii_4

Congreso Nacional. Ley de amparo laboral de la mujer. (2000).

Congreso Nacional. Ley de Maternidad Gratuita y Atención a la Infancia. Recuperado de https://www.igualdad.gob.ec/wp-

content/uploads/downloads/2017/11/ley_maternidad_gratuita _atencion_infancia.pdf

Consejo de Educación Superior. Ley Ogánica de Educación Superior LOES. (2018).

Consejo Nacional de Planificación. (2013). Plan Nacional para el Buen Vivir (Primera ed). Recuperado de www.planificacion.gob.ec

Consejo Universitario. Estatuto de la Universidad Laica Eloy Alfaro de Manabí. (2014).

Herdoíza, M. (2015). Construyendo Igualdad en la Educación Superior. Recuperado de http://www.educacionsuperior.gob.ec/wp-content/uploads/downloads/2015/10/349720UNESCO.pdf

Kabeer, N. (2001). Discussing Women's Empowerment: Theory and Practice. SIDA Studies, 136. Recuperado de www.sida.se

Luna, L. (1998). La representatividad del sujeto mujer en el feminismo de la Transición (C. Fagoada, ed.). Comunidad de Madrid.

Matarranz, M., \& Ramírez, E. (2018). Igualdad de género y Educación Superior: Retos por alcanzar en la Unión Europea. Archivos Analíticos de Políticas Educativas, 26(69). doi: 10.14507/epaa.26.2590

Melero, N. (2010). Reivindicar la igualdad de mujeres y hombres en la sociedad: una aproximación al concepto de género. Revista Castellano-Manchega de Ciencias Sociales, 11, 73-83. doi: 10.20932/barataria.v0i11.152

Naciones Unidas. (2015). Objetivos de Desarrollo del Milenio Informe de 2015. Naciones Unidas, 72. doi: $10.1108 / 17427370810932141$

Navarro, M., Ramos, G., \& Cejas, M. (2018). El derecho a la igualdad en el ámbito educativo: una perspectiva moderna para la inclusión de la mujer. Revista de Derecho, 7, 89-04. doi: 10.31207/ih.v7i0.171

Ordorika, I. (2015). Equidad de género en la Educación Superior. Revista de la Educación Superior, XLIV (174), 7-17. doi: 10.1016/j.resu.2015.06.001

Organización de las Naciones Unidas. (1995a). Declaración y Plataforma de Acción de Beijing. En La Cuarta Conferencia Mundial sobre la Mujer doi: 10.1017/CBO9781107415324.004

Organización de las Naciones Unidas. (1995b). Informe de la Cuarta Conferencia Mundial sobre la Mujer. Recuperado de https://www.un.org/womenwatch/daw/beijing/pdf/Beijing full report S.pdf 
Palacios, P. (2008). Los derechos de las mujeres en la nueva Constitución. Institut de Recherche et Débat Sur La Gouvernance. Retrieved from http://www.institutgouvernance.org/fr/analyse/fiche-analyse-452.html\#h8

Pérez Sedeño, E. (2018). Conocimiento y Educación Superior desde la perspectiva de género: sociología, políticas públicas y epistemología. Revista de estudios de la Ciencia y la Tecnología, 7(1), 121-142.

Peterson, H. (2017). Gender and prestige in Swedish academia: Exploring senior management in universities and university colleges. Scandinavian Journal of Educational Research, 61(1), 1-17. doi: 10.1080/00313831.2015.1066437

Reimberto, C. (2008). Los derechos de las mujeres en la Constitución de la República del Ecuador (Consejo $\mathrm{N}^{\mathrm{a}}$ ). Recuperado de https://core.ac.uk/download/pdf/143430038.pdf

Ruzzeddu, M. (2019). Women and Science Models of Participation. Advances in Gender and Cultural. Research in Business and Economics, 43-57. doi: 10.1007/978-3-030-00335-7

Tribunal Constitucional del Ecuador. Constitución de la República del Ecuador. Pub. L. №. Registro oficial $\mathrm{N}^{0}, 449$ (2008).

Zabludovsky, G. (2007). Las mujeres en México: trabajo, educación superior y esferas de poder. Política y Cultura, 28, 09-41. 